\title{
Anomalous Hall effect in MnAs: Intrinsic contribution due to Berry curvature
}

\author{
C. Helman $\odot,{ }^{1}$ A. Camjayi, ${ }^{2}$ E. Islam, ${ }^{3}$ M. Akabori,${ }^{3}$ L. Thevenard $\odot,{ }^{4}$ C. Gourdon, ${ }^{4}$ and M. Tortarolo ${ }^{5, *}$ \\ ${ }^{1}$ Instituto de Nanociencia y Nanotecnología, CONICET-CNEA, Centro Atómico Bariloche, Avenida Bustillo 9500, \\ 8400 S.C. de Bariloche, Rio Negro, Argentina \\ ${ }^{2}$ Departamento de Física, FCEyN, UBA and IFIBA, Conicet, Pabellón 1, Ciudad Universitaria, 1428 C.A.B.A., Argentina \\ ${ }^{3}$ Center for Nano Materials and Technology, Japan Advanced Institute of Science and Technology, \\ 1-1 Asahidai, Nomi, Ishikawa 923-1292, Japan \\ ${ }^{4}$ Institut des Nanosciences de Paris, Sorbonne Université, CNRS, UMR 7588, 4 place Jussieu, F-75005 Paris, France \\ ${ }^{5}$ Instituto de Nanociencia y Nanotecnología, CONICET-CNEA, Centro Atómico Constituyentes, Avenida General Paz 1499, \\ B1650 Villa Maipú, Buenos Aires, Argentina
}

(Received 30 November 2020; accepted 15 March 2021; published 7 April 2021)

\begin{abstract}
We present an experimental and theoretical study of the anomalous Hall effect (AHE) in MnAs epilayers grown over GaAs, with the aim to identify the intrinsic contribution to the AHE, which can be accurately evaluated using ab initio electronic structure calculations. Our magnetotransport measurements show a quadratic behavior of the Hall resistivity with longitudinal resistivity, characteristic of scattering-independent processes, thus enabling the comparison with our ab initio calculations. The calculated Berry phase contribution to the AHE is in quantitative agreement with the measured AHE in these epilayers. Moreover, the predicted anisotropic dependence of the experimental AHE on the magnetization is well reproduced.
\end{abstract}

DOI: 10.1103/PhysRevB.103.134408

\section{INTRODUCTION}

The anomalous Hall effect (AHE) has been a means to characterize the magnetization in spintronic devices composed of very thin magnetic layers, in which standard magnetometry measurements are not suited to the small magnetic volumes [1,2]. It can also explore magnetization dynamics, using rectifying effects [3]. Beyond being a characterization tool, the AHE has emerged as an experimental probe of the Berry phase [4]. It is characterized by the appearance of a spontaneous Hall current in an out-of-plane magnetized ferromagnet in response to an electric field [5]. Early theories explain it from extrinsic origins as skew scattering [6] and side jump [7] mechanisms due to the spin-orbit interaction (SOI). Nevertheless, during the last decade, several works on metallic ferromagnets with moderate conductivity reported an intrinsic contribution to the AHE (i.e., arising from the band structure of the perfect crystal) related to the Berry phase. This Berry phase gives rise to a transverse velocity of the electrons and hence to a Hall current [4].

Jungwirth et al. [8] presented a theory of the AHE in ferromagnetic (III, Mn) V semiconductors relating the anomalous Hall conductivity (AHC) in a homogeneous ferromagnet to the Berry phase. The quantitative agreement between theory and experiment in both (In,Mn)As and (Ga,Mn)As suggests that this disorder-independent contribution to the AHE dominates in these systems. Later, a series of theoretical and experimental works $[9,10]$ led to a unified theory of the AHE for ferromagnetic metals $[11,12]$, revealing a crossover from

*Corresponding author: tortarol@tandar.cnea.gov.ar extrinsic to intrinsic AHE behavior for increasing longitudinal conductivity. Later, Nagaosa et al. [13] proposed to classify the AHE in ferromagnets according to the following three empirical regimes:

(i) A bad-metal regime with $\sigma_{x x}<10^{4}(\Omega \mathrm{cm})^{-1}$, where the dependence of $\sigma_{x y}^{A H}$ on the longitudinal conductivity $\sigma_{x x}$ is well described experimentally by $\sigma_{x y}^{A H} \propto \sigma_{x x}^{1.6-1.8}$.

(ii) The intermediate region with $\sigma_{x x} \sim 10^{4}-10^{6}(\Omega \mathrm{cm})^{-1}$ such as in pure metals where $\left|\sigma_{x y}^{A H}\right|$ is fairly independent of $\sigma_{x x}$, i.e., of the scattering time, and thus possibly of intrinsic origin.

(iii) An extremely conducting case with $\sigma_{x x} \sim$ $10^{6}(\Omega \mathrm{cm})^{-1}$, where $\left|\sigma_{x y}^{A H}\right| \propto \sigma_{x x}$ is dominated by the skew scattering contribution.

Recently, Ma et al. [14] reported on the anisotropic Berry phase contribution to the AHE in MnAs. They obtained experimentally the intrinsic contribution to the AHE and ascribed the anisotropic effect to an anisotropic SOI in the Berry phase contribution but without the support from band structure calculations. The anisotropy of the band structure is usually reflected in the dependence of the intrinsic AHE on the magnetization orientation [15-17]. However, after studying both the influence of the current (electron momentum) and magnetization direction, $\mathrm{Ma}$ and coworkers reported that the Berry phase anisotropy is dominated by the current direction with a relatively small dependence on the magnetization orientation.

In addition, it has been demonstrated that when the Fermi energy lies within a SOI band splitting, the intrinsic AHC $\sigma_{x y}^{A H-i n t}$ is abruptly enhanced $[11,12]$, and this enhancement depends on the magnetization orientation by the magnetocrystalline anisotropy. To address these points, we show with experiments and theoretical calculations that the AHE in 


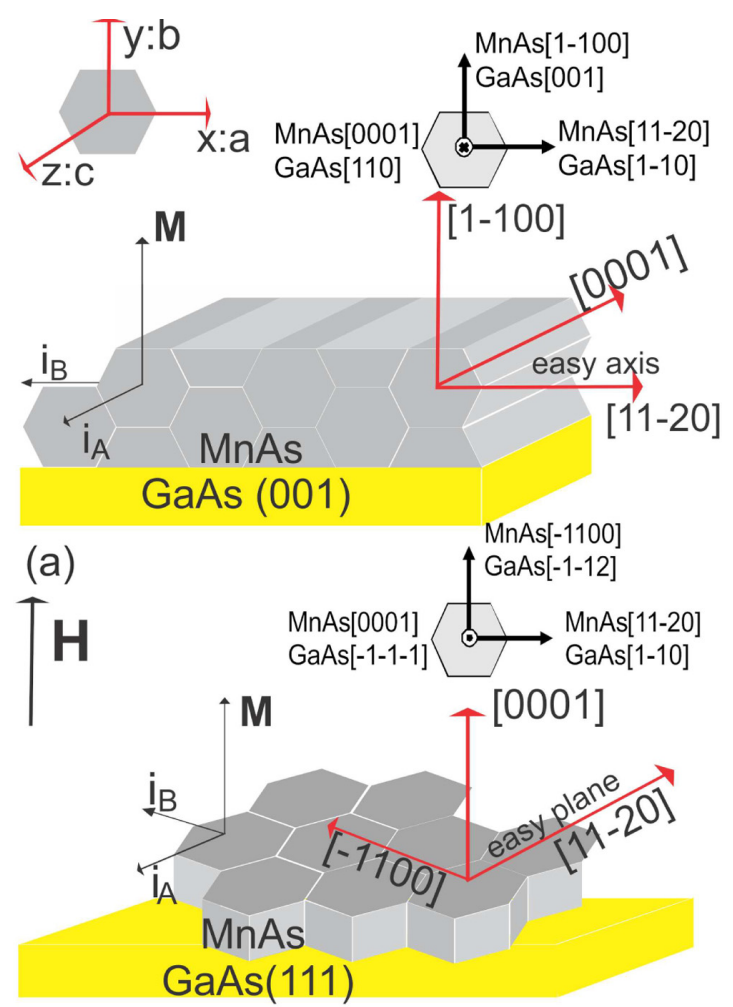

(b)

FIG. 1. Epitaxial orientation of the samples: (a) MnAs/GaAs001, with $i_{A} / /[0001], i_{B} / /[11-20]$, and (b) MnAs/GaAs-111, with $i_{A} / /[11-20]$ and $i_{B} / /[-1100] . i_{A}$ and $i_{B}$ indicate the orthogonal current directions used and were hence averaged to obtain the Hall magnetoresistance $\rho_{x y}$.

MnAs epilayers is dominated by the Berry phase contribution, and we analyze its dependence on the magnetization orientation by calculating the Berry curvature (BC) for relevant magnetization directions.

\section{SAMPLES AND EXPERIMENT}

Epitaxial MnAs films on GaAs single-crystalline substrates show the coexistence of a ferromagnetic hexagonal $\alpha$ phase and a paramagnetic distorted orthorhombic $\beta$ phase around room temperature, from 250 to $325 \mathrm{~K}$, depending on growth conditions, substrate orientation, and sample thickness [18-22]. Below $250 \mathrm{~K}$ samples are completely in the $\alpha$ ferromagnetic phase, and above $325 \mathrm{~K}$, they are in the paramagnetic $\beta$ phase. Two different MnAs films were grown by molecular beam epitaxy on $\mathrm{GaAs}(001)$ and $\mathrm{GaAs}(111) \mathrm{B}$, as depicted in Fig. 1. Samples will subsequently be named MnAs-001 and MnAs-111, with their thicknesses being 33 and $15 \mathrm{~nm}$, respectively. Above $6 \mathrm{~nm}$ Ma et al. [14] suggested that the Berry phase conductivity $\sigma^{\mathrm{AH}-\mathrm{int}}$ attains a constant value independent of the thickness, allowing us to compare the transport properties of both samples. Sample growth and characterization are reported in Refs. [23,24]. Sample MnAs-111 shows an isotropic magnetic behavior in plane and a hard magnetic axis along [0001]. Sample MnAs-001 has an easy magnetic axis along the [11-20] direction, an intermediate anisotropy axis along [1-100], and a hard one along [0001].

Magnetotransport data and magnetization measurements as a function of external magnetic field and temperature were done in a Quantum Design physical property measurement system using the Van der Pauw electric contact configuration [25]. Applying the field perpendicular to the plane allows us to study the magnetotransport properties when the magnetization is oriented parallel to the intermediate anisotropy [1-100] axis [Fig. 1(a)] and parallel to the hard anisotropy [0001] axis [Fig. 1(b)]. Previous works [14,26] reported that while the magnetic properties are anisotropic in the plane of the epilayers, the electronic transport properties within the plane show negligible anisotropy, which allows us to average the longitudinal resistivity $\left[\rho_{x x A B}=\left(\rho_{x x A}+\rho_{x x B}\right) / 2\right]$ and the transversal (Hall) resistivity $\left[\rho_{x y A B}=\left(\rho_{x y A}+\rho_{x y B}\right) / 2\right]$ for two different $\left(i_{A}, i_{B}\right)$ current directions (indicated in Fig. 1) as performed in Van der Pauw samples. We corroborated that both $\left(\rho_{x x A}, \rho_{x x B}\right)$ and $\left(\rho_{x y A}, \rho_{x y B}\right)$ pairs are within $10 \%$ agreement before averaging.

In order to study the main characteristics of the AHE in MnAs/GaAs a series of transport measurements was done between 5 and $300 \mathrm{~K}$ with an applied magnetic field up to $9 \mathrm{~T}$ perpendicular to the sample surface. The temperature dependence of the longitudinal resistivity $\rho_{x x}$ at $H=0$ for both samples [insets of Figs. 2(c) and 2(f), respectively] exhibits a metallic behavior, with the resistivity $\rho_{x x}$ decreasing monotonically with decreasing temperature. Figure 2(a) shows the temperature variation of the magnetoresistance, $\mathrm{MR}=\left[\rho_{x x}(H)-\rho_{x x}(0)\right] / \rho_{x x}(0)$, for sample MnAs-001 in the $50-250 \mathrm{~K}$ range. We note a change in the high-field MR from positive to negative around $T \sim 80 \mathrm{~K}$, with a weak dependence on the applied field around this temperature. The MnAs-111 sample shows negative MR in the whole temperature range, as displayed in Fig. 2(d). These MR features of MnAs were already presented in [26-28], showing mainly the same results, and will not be further discussed in the present work.

The Hall resistivity in a ferromagnet can be expressed as

$$
\rho_{\text {Hall }}=R_{H} H+R_{A} M(T, H),
$$

where $R_{H}$ is the ordinary Hall coefficient, $H$ is the external field, $R_{A}$ is the anomalous (or extraordinary) Hall coefficient, and $M(T, H)$ is the sample magnetization in the out-of-plane direction. The second term is the spontaneous magnetization contribution to the Hall effect [29]. Several mechanisms were proposed in the literature to describe this last term, such as skew scattering, side jump, and an intrinsic mechanism related to the electronic band structure, Berry curvature [4,6,7].

Figure 2(b) displays $\rho_{x y}$ in MnAs-001. It is dominated by the anomalous Hall effect up to the saturation field of the magnetization $(\sim 1 \mathrm{~T})$, where the ordinary Hall contribution appears as a linear behavior with the applied field. For MnAs-111 the same behavior is observed in the Hall magnetoresistivity versus field [Fig. 2(e)], albeit with a larger saturation field $(\approx 4 \mathrm{~T})$. This larger field is consistent with the magnetic anisotropy of MnAs [30]. The field is applied 

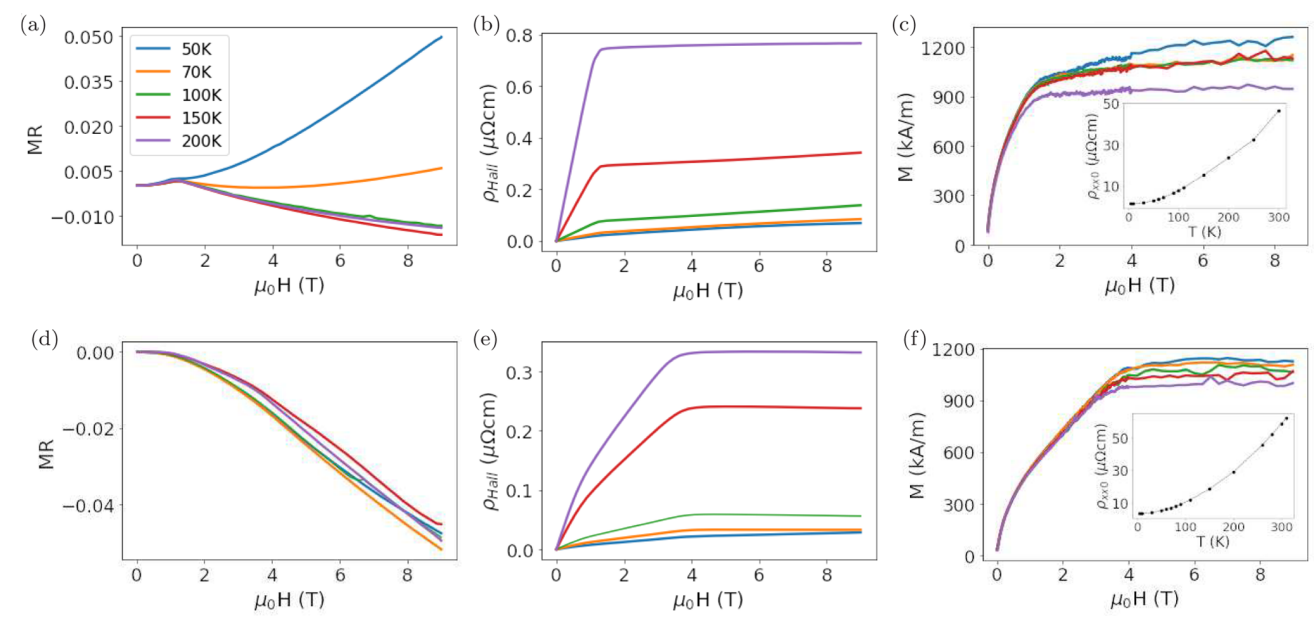

FIG. 2. Top: MnAs-001 sample [see Fig. 1(a)]. (a) Magnetoresistance, (b) Hall magnetoresistivity, and (c) out-of-plane magnetization (inset: longitudinal resistivity at $\mu_{0} H=0$ ). Bottom: MnAs-111 sample [see Fig. 1(b)]. (d) Magnetoresistance, (e) Hall magnetoresistivity, and (f) out-of-plane magnetization (inset: longitudinal resistivity at $\mu_{0} H=0$ ).

along the $c$ hard axis for MnAs-111 and along an intermediate anisotropy axis for MnAs-001.

We restrict our analysis to the temperature range between 50 and $200 \mathrm{~K}$, on the one hand, to avoid anomalies of the Hall magnetoresistance at low temperatures, where a change in the sign of $R_{H}$ was ascribed to the presence of positive and negative carriers [26], and, on the other hand, to avoid the coexistence of ferromagnetic and paramagnetic phases at higher temperatures. The anomalous Hall resistivity $\rho^{A H}$ is obtained as the zero-field extrapolation of the high-field linear $\rho_{x y}(H)$ data. It is presented in Fig. 3 for both MnAs-001 and MnAs-111 samples.

For each temperature we calculate the AHC $\left|\sigma_{x y}^{A H}(T)\right|$ as a function of the longitudinal conductivity $\sigma_{x x}(T)$ using the tensorial relations $\left|\sigma_{x y}^{A H}\right| \approx \rho_{x y}^{A H} / \rho_{x x}^{2}$ and $\sigma_{x x} \approx 1 / \rho_{x x}$. Figure 3(b) shows that $\left|\sigma_{x y}^{A H}\right|$ is nearly constant for sample MnAs-100 $\left[\sim 1300 \pm 100(\Omega \mathrm{cm})^{-1}\right]$. The same is found for sample MnAs-111 with a smaller value $[\sim 750 \pm$ $100(\Omega \mathrm{cm})^{-1}$ ]. Note that in Fig. 3(b) we use the same scale as in Ref. [10] to highlight the comparison with those experiments, done in a wider resistivity range of 5 orders of magnitude. A constant $\sigma_{x y}^{A H}$ means that $\rho_{x y}^{A H} \propto \rho_{x x}^{2}$. This is confirmed independently by the linear fit of $\rho_{x y}^{A H}$ vs $\rho_{x x}^{2}$ shown in Fig. 3(c),

$$
\rho_{x y}^{A H}=A+B \rho_{x x}^{2},
$$

where the first term is found to be negligible compared to the second one $\left(A \sim 10^{-9}, B \sim 10^{3}\right)$ for all values of $\rho_{x x}(T)^{2}$ with $T>50 \mathrm{~K}$. We now examine these results in the light of the scaling of the anomalous Hall resistivity proposed in Refs. [14,31], $\rho_{x y}^{A H}=a \rho_{x x 0}+b \rho_{x x 0}^{2}+\sigma^{A H-\text { int }} \rho_{x x}^{2}$, where $\rho_{x x 0}$ is the $T=0$ longitudinal resistivity, $a$ is the skew scattering contribution, $b$ is the side jump one, and $\sigma^{A H-\text { int }}$ is the strength of the Berry phase related intrinsic AHC. From the results of Figs. 3(b) and 3(c) discussed above we can thus safely identify our experimental $\sigma^{A H}$ with the intrinsic contribution $\sigma^{A H-\text { int }}$. We conclude that in the range of temperature investigated here the intrinsic contribution to the AHE dominates. In order to strengthen this conclusion by a quantitative comparison we will now obtain theoretically the intrinsic contribution to the AHC by calculating the Berry phase contribution. (a)

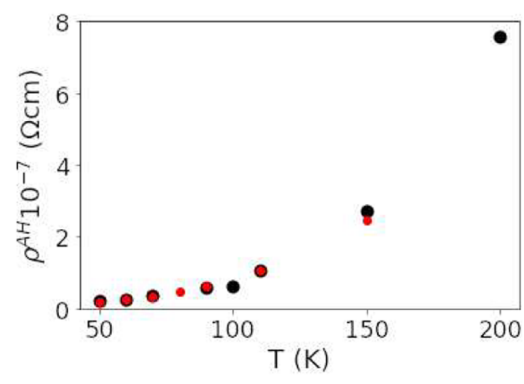

(b)

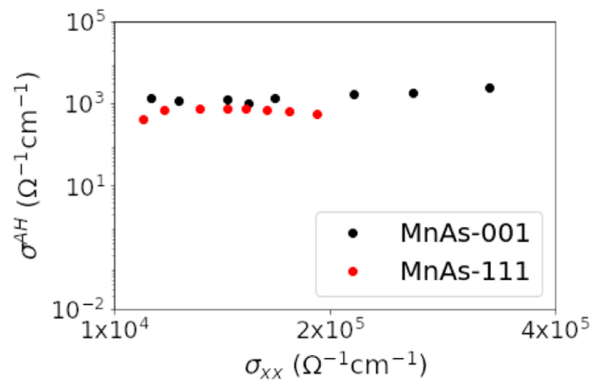

(c)

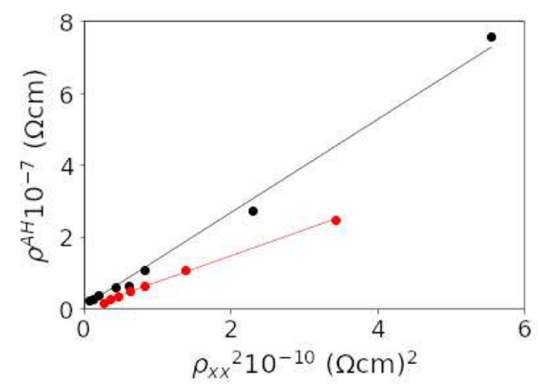

FIG. 3. (a) Anomalous Hall resistivity versus temperature, (b) anomalous Hall conductivity as a function of the longitudinal resistivity extracted from data between 50 and $200 \mathrm{~K}$, and (c) linear fit of the anomalous Hall resistivity versus the square longitudinal resistivity. The fact that $\left|\sigma^{A H}\right| \sim$ const in (b) comes from the quadratic dependence of $\rho_{x y}^{A H}$ on $\rho_{x x}$ displayed in (c). 

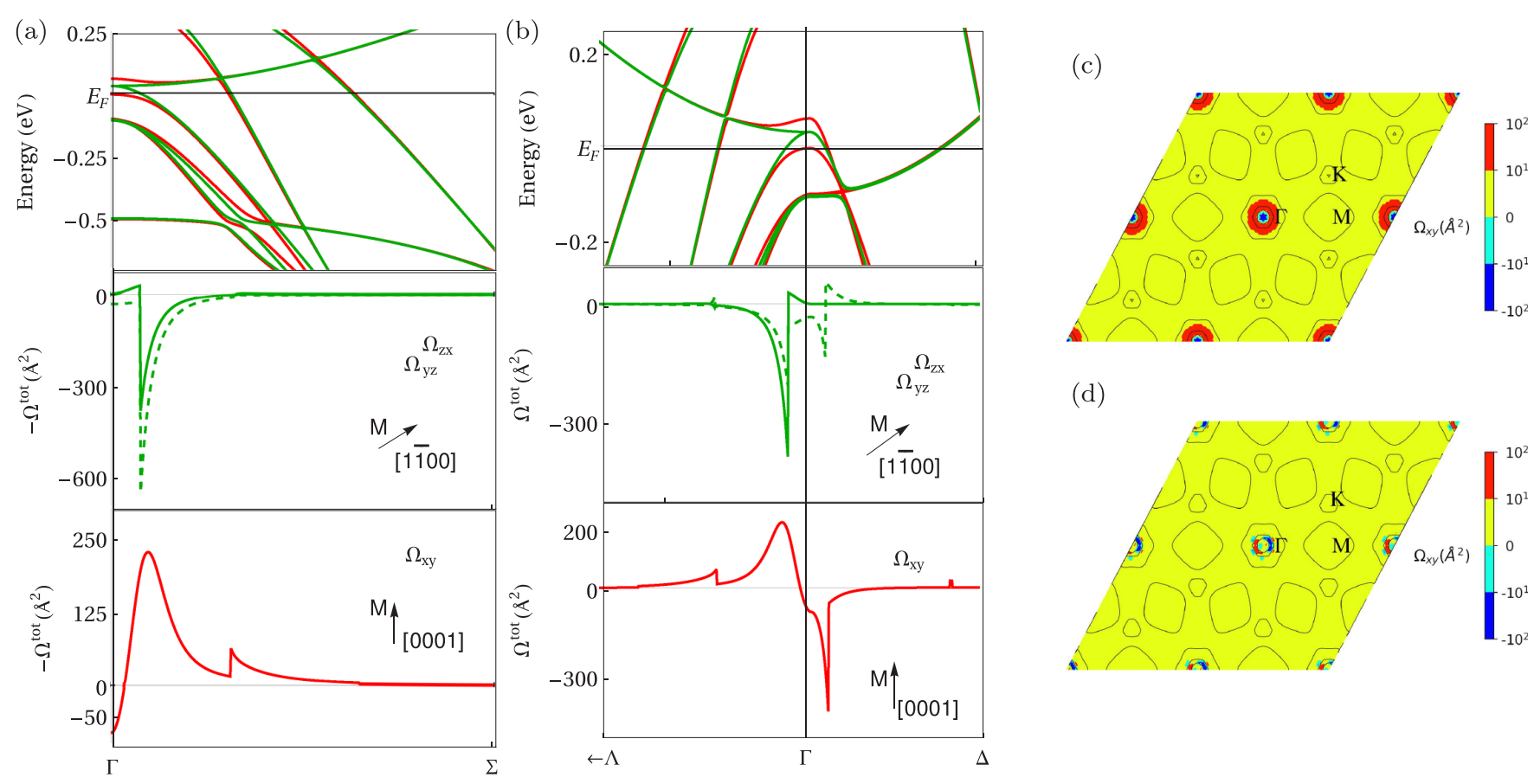

FIG. 4. (a) and (b) The first row shows the detail of the band structure at specific points of the Brillouin zone close to the Fermi level $\varepsilon_{F}$. Second and third rows correspond to the components of the $\mathrm{BC} \Omega_{\alpha \beta}(\mathbf{k})$ with the magnetization oriented along the [1-100] orientation (green lines) and [0001] orientation (red lines), respectively. Color map of the Berry curvature tensor corresponding to the magnetization saturated in (c) the [0001] direction and (d) [1-100] direction. Black lines are the Fermi surface intersection with the (001) plane.

\section{THEORETICAL ANALYSIS OF THE INTRINSIC AHE}

The Berry phase contribution to $\sigma^{A H-\text { int }}$ in a magnetic material with a perfect crystal structure comes from features of the electronic band structure and, being scattering independent, can be evaluated using $a b$ initio methods. Specifically, the Berry phase of Bloch electrons is related to $\sigma^{A H-i n t}$ by Berry curvature $\Omega_{\alpha \beta}(\mathbf{k})$. Coming from the Kubo formula derivation, Berry curvature can be expressed as $[9,32,38]$

$$
\Omega_{\alpha \beta}(\mathbf{k})=\sum_{n \neq m}\left(f_{n}-f_{m}\right) \frac{\hbar^{2} \operatorname{Im}\left\langle\psi_{n}\left|v_{\alpha}\right| \psi_{m}\right\rangle\left\langle\psi_{m}\left|v_{\beta}\right| \psi_{n}\right\rangle}{\left(\varepsilon_{m}-\varepsilon_{n}\right)^{2}}
$$

where $f_{n}$ is the Fermi-Dirac distribution function, $\psi_{n}$ are the eigenstates of the Bloch electrons, and indices $n, m$ are band labels. At $T=0$ only occupied states contribute to the summation.

The velocities $v_{i}=\hbar^{-1} \partial_{k_{i}} \varepsilon_{n, \mathbf{k}}$ and the energy levels $\varepsilon_{n, \mathbf{k}}$ are obtained employing the QUANTUM ESPRESSO code [33] in conjunction with maximally localized Wannier function techniques. See the Appendix for details.

Finally, the anomalous Hall conductivity is calculated as

$$
\sigma_{\alpha, \beta}^{\mathrm{AHE}}=-\frac{e^{2}}{\hbar} \int_{B Z} \frac{d \mathbf{k}}{(2 \pi)^{3}} \Omega_{\alpha \beta}
$$

The $\mathrm{BC}$ has strong variations in reciprocal space, making a very dense mesh necessary to achieve the convergence of Eq. (4) [9]. To capture the fine details of the Berry curvature (Fig. 4), we used an adaptive uniform mesh refinement of $10^{3}$ points around special points where the absolute value of the curvature is greater than unity. These special points with their mesh refinement represent more than $70 \%$ of the total points where we evaluate the Berry curvature. Convergence was achieved with $\sim 3 \times 10^{6}$ points in reciprocal space.

Since SOI is symmetry breaking, different magnetization orientations are not equivalent. Calculations were made with the magnetization parallel to the [1-100] and [0001] crystal directions, corresponding to the measurement geometry at saturation for samples MnAs-001 and MnAs-111, respectively.

Figure 4 shows details of the band structure (top row) around the high-symmetry point $\Gamma$. In the middle row, $\Omega_{z x}(\mathbf{k})$ (green dashed lines) and $\Omega_{y z}(\mathbf{k})$ (green solid lines) are displayed for the MnAs-001 sample configuration, with the magnetization oriented in the [1-100] direction. The bottom row shows $\Omega_{x y}(\mathbf{k})$ (red line) for the magnetization oriented in the [0001] direction, which is the experimental geometry of the MnAs-111 sample. The full band structure Fig. 5(b) and the high-symmetry points [Fig. 5(a)] are displayed in the Appendix.

All three components of the $\mathrm{BC}$ are negligible in most of the momentum space except near $\Gamma$, where the bands showing degeneracy or band splitting due to the SOI (commonly referred to as band splitting [34]) lead to peaks in the BC. Notice that the $m, n$ bands contribute only to the $\mathrm{BC}$ on the $\mathbf{k}$ points where they have different occupations.

When the magnetization is oriented along the [0001] direction, the band degeneracy in $\Gamma$ is lifted by the SOI, leading to a small energy gap that gives a small denominator in Eq. (3), making $\Omega_{\alpha \beta}(\mathbf{k})$ very large in a small interval. Also, there is a peak in the $\Gamma$ to $\Delta$ path when the band crosses $E_{F}$. On the other hand, when the magnetization is saturated along [1-100], there is a peak in $\Gamma$ due to the band (near) degeneracy. These large peaks act as magnetic monopoles in $k$ space [35] and can be seen in $\Omega_{\alpha \beta}(\mathbf{k})$ [Figs. 4(c) and 4(d)] in regions where 
TABLE I. Intrinsic AHE conductivity: Experimental and calculated values.

\begin{tabular}{lcc}
\hline \hline Sample & $\sigma_{\text {exp }}^{A H-\text { int }}(\Omega \mathrm{cm})^{-1}$ & $\sigma_{\text {calc }}^{A H-\text { int }}(\Omega \mathrm{cm})^{-1}$ \\
\hline MnAs-001 & $1300 \pm 100$ & $1400 \pm 100$ \\
MnAs-111 & $740 \pm 100$ & $780 \pm 100$ \\
\hline \hline
\end{tabular}

the Fermi lines [intersection of the Fermi surface with (001)] have (near) degeneracy or band splittings due to SOI.

As $\sigma^{A H-\text { int }}$ [Eq. (2)] is the integral of $\Omega_{\alpha \beta}(\mathbf{k})$, when $E_{F}$ lies within the SOI gap $\left(\Delta_{\text {SOI }}\right)$ or close to a band degeneracy, $\sigma^{A H-\text { int }}$ reaches a maximum due to the peaks in $\Omega_{\alpha \beta}(\mathbf{k})$. If both bands are empty $\left(E_{F}<-\Delta_{\text {SOI }}\right)$ or occupied $\left(E_{F}>\right.$ $\Delta_{\text {SOI }}$ ), their $f_{n, m}$ are equal, and their contributions are canceled in Eq. (3). Only when $E_{F}$ lies within $\Delta_{\text {SOI }}$ or a (near) band degeneracy is the contribution due to the $\mathrm{BC}$ maximal and $\sigma^{A H-\text { int }}$ "resonantly" enhanced $[11,12]$.

\section{DISCUSSION}

According to the classification proposed by Nagaosa et al. [13], our samples with $\sigma_{x x}=\rho_{x x}^{-1}=10^{5}(\Omega \mathrm{cm})^{-1}$ fall into the so-called good metal regime with $10^{4}<\sigma_{x x}<$ $10^{6}(\Omega \mathrm{cm})^{-1}$, which is an intrinsic or scattering-independent regime where $\sigma^{A H}$ is roughly independent of $\sigma_{x x}$, as can be seen in our data analysis [Fig. 3(b)].

The fact that $\rho^{A H} \propto \rho_{x x}^{2}$ suggests that the extrinsic skew scattering contribution $\left(\rho_{x y} \propto \rho_{x x}\right)$ has already decayed in this regime. In addition, the agreement of the experimental and calculated values (Table I) enables the association of the plateau region in our data with a regime dominated by the intrinsic Berry phase contribution. The values reported in this work for $\left|\sigma^{A H-\text { int }}\right|$ are in agreement with the experimental ones reported for pure metals like $\mathrm{Fe}, \mathrm{Co}$, and Gd [10] and are also consistent with $a b$ initio calculations for bcc Fe [9]. Nevertheless, our values are higher than the ones reported in Ref. [14], which carried out a similar study on MnAs/GaAs. We can say that the agreement between our experiments and theoretical calculations supports our results. Moreover, our experimental measurements and $a b$ initio calculations show that the system is fulfilling the conditions for the AHE to be dominated by the intrinsic contribution, as discussed by Onoda et al. [11,12]: (i) We showed that the Berry phase contribution to the AHC is strong when the $E_{F}$ level lies on a splitting of the band structure due to the SOI or near (nearly) degenerate bands (Fig. 4). (ii) Both experimental and calculated $\sigma_{x y}^{A H}$ are approximately $10^{-3}(\Omega \mathrm{cm})^{-1}$, and (iii) the longitudinal resistivity of our samples is within $\rho_{x x} \sim$ $1-10 \mu \Omega \mathrm{cm}$. Within these conditions the ab initio calculations give accurate values of $\sigma^{A H-\text { int }}$, suggesting the AHE can, indeed, be associated with the Berry phase contribution. Moreover, our results show the dependence of $\sigma^{A H-\text { int }}$ on the magnetization direction, as the results summarized in Table I show that for both experiments and theory the intrinsic AHE is larger when the magnetization is aligned along the intermediate easy axis than along the hard one. These are consistent results, as both the AHE and the magnetocrystalline anisotropy have the same origin in the SOI.

\section{CONCLUSION}

We have experimentally extracted the AHC in MnAs epilayers and theoretically calculated its intrinsic contribution due to the $\mathrm{BC}$ of the dispersion bands. Our measurements show a quadratic dependence of the anomalous resistivity on the longitudinal resistivity $\left(\rho^{A H} \propto \rho_{x x}^{2}\right)$ which is characteristic of scattering-independent processes, allowing us to compare them with our $a b$ initio calculations. The experimental results and the theoretically calculated intrinsic conductivities show good agreement, which makes it possible to attribute the measured AHC in MnAs to the Berry phase related contribution (intrinsic) to the anomalous conductivity $\sigma^{\mathrm{AH}-\mathrm{int}}$. These results contribute to the experimental evidence of the Berry phase mechanism in metals and could span the possibility of band structure engineering of topological transport in MnAs epilayers, provided the scattering-independent contribution to the AHE is more controllable and reproducible than the scattering-dependent one.

\section{ACKNOWLEDGMENTS}

We acknowledge D. Hrabovsky and Y. Klein for their assistance in the magnetotransport measurements at the Plateforme de mesures physiques à basse température of Sorbonne Université. We also thank V. Ferrari for her comments on the manuscript. This work was done under LIFAN

(a)

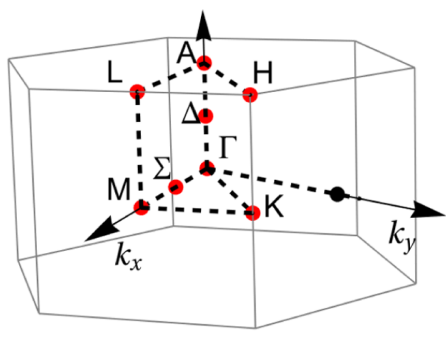

(b)

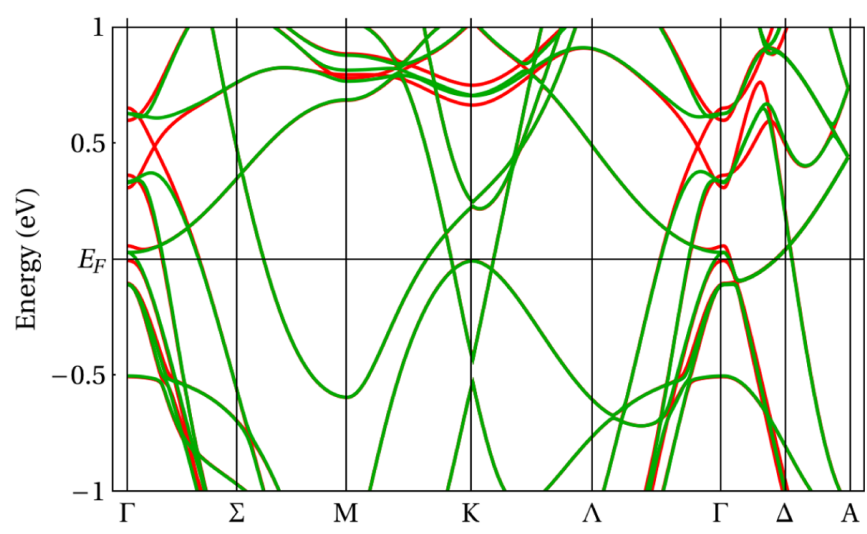

FIG. 5. (a) High-symmetry points for the $P 63 / \mathrm{mmc}$ space group. (b) Band structure calculated including the SOI along the path of high-symmetry points shown in (a). The green and red lines correspond to magnetization saturated along the [1-100] and [0001] orientations, respectively. In this energy window, the energy bands with the magnetization saturated along [1-100] (green lines) considering the SOI overlap the spin-polarized calculation without SOI in this energy range. 
(Laboratoire International Franco Argentin en Nanosciences) collaboration. M.T. had partial support from the CONICET research stay for assistant researchers program and PIP Grant No. 11220115-0100213 CO. A.C. gratefully acknowledges support from CONICET and ANPCyT.

\section{APPENDIX: BAND STRUCTURE}

Our calculations are done considering the MnAs to be in the ferromagnetic $\alpha$ phase, which crystallizes for temperatures lower than $300 \mathrm{~K}$ in the hexagonal NiAs structure within the $P 63 / m m c$ space group, with unit cell parameters $a=3.73 \AA$ and $c / a=1.53$ [36]. We use the QUANTUM ESPRESSO code [34] in conjunction with the maximally localized Wannier function techniques implemented in WANNIER90 [37].
Calculations in QUANTUM ESPRESSOwere performed by using the projector augmented wave method with a fully relativistic pseudopotential for each atom ( $\mathrm{Mn}$ and As), with the generalized gradient approximation as the exchangecorrelation potential and SOI implemented in a nonlinear approximation. For the electronic calculation (including SOI), the convergence is achieved with a cutoff energy of $50 \mathrm{Ry}$ for the plane waves and a $20 \times 20 \times 16$ mesh in reciprocal space.

Figure 5(b) shows the band structure along a path of highsymmetry points [Fig. 5(a)], taking into account the SOI in the calculations. The magnetization is saturated along the [0001] direction [Fig. 5(b), red lines] and along the [1-100] direction [Fig. 5(b), green lines]. For this last case, the band structure fully overlaps with the spin-polarized calculation without taking into account the SOI, within the presented energy range.
[1] H. Ohno, D. Chiba, F. Matsukura, T. Omiya, E. Abe, T. Dietl, Y. Ohno, and K. Ohtani, Nature (London) 408, 944 (2000).

[2] D. Chiba, A. Werpachowska, M. Endo, Y. Nishitani, F. Matsukura, T. Dietl, and H. Ohno, Phys. Rev. Lett. 104, 106601 (2010).

[3] A. Yamaguchi, K. Motoi, A. Hirohata, and H. Miyajima, Phys. Rev. B 79, 224409 (2009).

[4] D. Xiao, M.-C. Chang, and Q. Niu, Rev. Mod. Phys. 82, 1959 (2010).

[5] Commemorative Symposium on the Hall Effect and Its Applications, edited by C. L. Chien and C. R. Westgate (Plenum, New York, 1980).

[6] J. Smit, Physica (Amsterdam) 21, 877 (1955); 24, 39 (1958).

[7] L. Berger, Phys. Rev. B 2, 4559 (1970).

[8] T. Jungwirth, Q. Niu, and A. H. MacDonald, Phys. Rev. Lett. 88, 207208 (2002).

[9] Y. Yao, L. Kleinman, A. H. MacDonald, J. Sinova, T. Jungwirth, D. S. Wang, E. Wang, and Q. Niu, Phys. Rev. Lett. 92, 037204 (2004).

[10] T. Miyasato, N. Abe, T. Fujii, A. Asamitsu, S. Onoda, Y. Onose, N. Nagaosa, and Y. Tokura, Phys. Rev. Lett. 99, 086602 (2007).

[11] S. Onoda, N. Sugimoto, and N. Nagaosa, Phys. Rev. Lett. 97, 126602 (2006).

[12] S. Onoda, N. Sugimoto, and N. Nagaosa, Phys. Rev. B 77, 165103 (2008).

[13] N. Nagaosa, J. Sinova, S. Onoda, A. H. MacDonald, and N. P. Ong, Rev. Mod. Phys. 82, 1539 (2010).

[14] J. L. Ma, H. L. Wang, X. L. Wang, and J. H. Zhao, Phys. Rev. B 97, 064402 (2018).

[15] L. Wu, Y. F. Li, J. L. Xu, D. Z. Hou, and X. F. Jin, Phys. Rev. B 87, 155307 (2013).

[16] D. Z. Hou, Y. F. Li, D. H. Wei, D. Tian, L. Wu, and X. F. Jin, J. Phys.: Condens. Matter 24, 482001 (2012).

[17] J. Stankiewicz and K. P. Skokov, Phys. Rev. B 78, 214435 (2008).

[18] L. Däweritz, F. Schippan, A. Trampert, M. Kästner, G. Behme, Z. M. Wang, M. Moreno, P. Schützendübe, and K. H. Ploog, J. Cryst. Growth 227-228, 834 (2001).
[19] A. K. Das, C. Pampuch, A. Ney, T. Hesjedal, L. Däweritz, R. Koch, and K. H. Ploog, Phys. Rev. Lett. 91, 087203 (2003).

[20] F. Iikawa, P. V. Santos, M. Kästner, F. Schippan, and L. Däweritz, Phys. Rev. B 65, 205328 (2002).

[21] V. M. Kaganer, B. Jenichen, F. Schippan, W. Braun, L. Däweritz, and K. H. Ploog, Phys. Rev. B 66, 045305 (2002).

[22] N. Mattoso, M. Eddrief, J. Varalda, A. Ouerghi, D. Demaille, V. H. Etgens, and Y. Garreau, Phys. Rev. B 70, 115324 (2004).

[23] M. E. Islam, M. Akabori, J. Cryst. Growth 463, 86 (2017).

[24] M. E. Islam and M. Akabori, Phys. B (Amsterdam, Neth.) 532, 95 (2018).

[25] L. J. van der Pauw, Philips Res. Rep. 13, 1 (1958).

[26] J. J. Berry, S. J. Potashnik, S. H. Chun, K. C. Ku, P. Schiffer, and N. Samarth, Phys. Rev. B 64, 052408 (2001).

[27] K.-J. Friedland, M. Kästner, and L. Däweritz, Phys. Rev. B 67, 113301 (2003).

[28] C. Helman, J. Milano, L. Steren, and A. M. Llois, J. Magn. Magn. Mater. 320, e415 (2008).

[29] E. M. Pugh and T. W. Lippert, Phys. Rev. 42, 709 (1932).

[30] L. B. Steren, J. Milano, V. Garcia, M. Marangolo, M. Eddrief, and V. H. Etgens, Phys. Rev. B 74, 144402 (2006).

[31] Y. Tian, L. Ye, and X. Jin, Phys. Rev. Lett. 103, 087206 (2009).

[32] X. Wang, J. R. Yates, I. Souza, and D. Vanderbilt, Phys. Rev. B 74, 195118 (2006).

[33] P. Giannozzi et al., J. Phys.: Condens. Matter 29, 465901 (2017).

[34] N. W. Ashcroft and N. D. Mermin, Solid State Physics (Saunders, New York, 1976), Chap. 9.

[35] Z. Fang, N. Nagaosa, K. S. Takahashi, A. Asamitsu, R. Mathieu, T. Ogasawara, H. Yamada, M. Kawasaki, Y. Tokura, and K. Terakura, Science 302, 92 (2003).

[36] I. Rungger and S. Sanvito, Phys. Rev. B 74, 024429 (2006).

[37] G. Pizzi et al., J. Phys.: Condens. Matter 32, 165902 (2020).

[38] M. G. Lopez, D. Vanderbilt, T. Thonhauser, and I. Souza, Phys. Rev. B 85, 014435 (2012). 